\title{
Residence-time distributions for chaotic flows in pipes
}

\author{
Igor Mezić \\ Department of Mechanical and Environmental Engineering, and Department of Mathematics, \\ University of California, Santa Barbara, California 93106-5070 \\ Stephen Wiggins \\ Control and Dynamical Systems 107-81, Caltech, Pasadena, California 91125 \\ David Betz \\ Department of Mechanical and Environmental Engineering, University of California, \\ Santa Barbara, California 93106-5070
}

(Received 13 April 1998; accepted for publication 30 December 1998)

\begin{abstract}
In this paper we derive two rigorous properties of residence-time distributions for flows in pipes and mixers motivated by computational results of Khakhar et al. [Chem. Eng. Sci. 42, 2909 (1987)], using some concepts from ergodic theory. First, a curious similarity between the isoresidence-time plots and Poincare maps of the flow observed in Khakhar et al. is resolved. It is shown that in long pipes and mixers, Poincaré maps can serve as a useful guide in the analysis of isoresidence-time plots, but the two are not equivalent. In particular, for long devices isoresidence-time sets are composed of orbits of the Poincare map, but each isoresidence-time set can be comprised of many orbits. Second, we explain the origin of multimodal residence-time distributions for nondiffusive motion of particles in pipes and mixers. It is shown that chaotic regions in the Poincare map contribute peaks to the appropriately defined and rescaled axial distribution functions. (C) 1999 American Institute of Physics. [S1054-1500(99)02401-5]
\end{abstract}

\begin{abstract}
Residence-time distributions have been in use as diagnostic tools for mixing in Chemical Engineering devices for a long time. In the past 15 years, a theory of mixing in laminar flows, Chaotic Advection, has been developed, with one of the most important diagnostic tools being Poincaré maps. In this paper we analyze the similarities and differences between these two concepts, using ergodic theory invoked by the link between the residence times and average velocities along particle paths. The residence-time plots contain information about the axial motion, while the Poincaré maps contain more information about the cross-sectional motion, and essentially no information about the axial motion. We also establish two different mechanisms for the multimodality of finite-time residence-time distributions observed in earlier works.
\end{abstract}

\section{INTRODUCTION}

In this paper we derive two rigorous properties of residence-time distributions for laminar flows in pipes and mixers motivated by computational results of Khakhar et al. ${ }^{1}$ Residence times have been in use for a long time as an important diagnostic tool in a number of engineering devices; see, e.g., Refs. 2-6. Poincaré maps, on the other hand, have been used for studying dynamics of particles in laminar fluid flows since the beginning of the chaotic advection studies. ${ }^{5,7}$ In the present work we show that these two methods are, in long devices, related via time averages of the velocity in the direction of the axis of the pipe. Using this observation, we discuss the similarity of residence-time plots and Poincare maps observed in Ref. 1 . We show that a set in the crosssection of the pipe on which the residence times are constant is composed of the orbit of the Poincare map. Many orbits can be within one such set. Thus in some sense Poincaré maps carry more information than residence-time plots. On the other hand, Poincaré maps do not contain any information about the axial motion, while residence-time plots (or, equivalently as we show below, velocity time-average plots) do. In addition, and again using the time averages of the velocity, we discuss the distribution of particles along the axis of the pipe, and residence-time distributions for chaotic flows in pipes. It is shown that chaotic zones in the Poincare map of the flow contribute peaks to the particle probability density and residence-time probability density.

\section{STEADY, PERIODIC FLOWS IN PIPES}

\section{A. Residence times}

Khakhar et al. ${ }^{1}$ considered a mixing device known as the partitioned-pipe mixer. It is a continuous flow device consisting of a pipe partitioned into a sequence of semi-circular ducts by means of rectangular plates positioned orthogonally with respect to each other; see Fig. 1. We refer to a length of pipe containing two plates as one "element" of the mixer. Hence, the mixer can be viewed as a number of elements placed end-to-end. We denote the total length of the pipe by $L$ and the length of its basic element by $l$. Note that $L / l$ is an integer.

The fluid to be mixed is forced through the pipe by means of an axial pressure gradient while the pipe is rotated about its axis relative to the plates. The mixing device is typically operated under creeping flow conditions, and the flow is steady. 


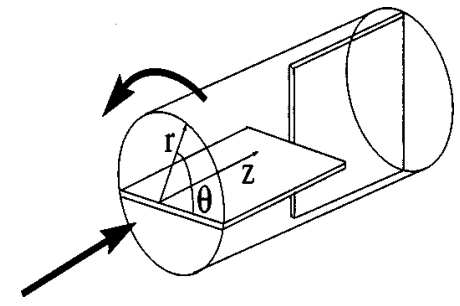

FIG. 1. Schematic of one element of the partitioned-pipe mixer.

Khakhar et al. ${ }^{1}$ have calculated the flow through the basic cell in the Stokes approximation to be

$$
\begin{aligned}
v_{r}=\frac{d r}{d t}= & \beta r\left(1-r^{\nu}\right) \sin (2 \theta), \\
\frac{v_{\theta}}{r}=\frac{d \theta}{d t}= & -\beta\left[2-(2+\nu) r^{\nu}\right] \sin ^{2} \theta, \\
v_{z}=\frac{d z}{d t}= & \frac{16 \pi}{\pi^{2}-8} \sum_{k=1}^{3}\left(r^{2 k-1}-r^{2}\right) \\
& \times \frac{\sin [(2 k-1) \theta]}{(2 k-1)\left[4-(2 k-1)^{2}\right]},
\end{aligned}
$$

where $\nu=\sqrt{11 / 3}-1$ and $\beta$ are constants, and $\beta$ is related to the speed of rotation of the wall of the pipe. In computations, the above flow field is used in the first half of the basic cell, and then rotated by 90 degrees and used for the second part of the cell. The assumptions used in the model are not physically accurate. However, the phenomenology of the chaotic flow-the existence of the isolated islands and chaotic zones-stays the same even if a careful numerical simulation of a realistic mixer is performed. ${ }^{8,9}$ Our analysis is theoretical and numerical computation is used only to illustrate the theory, so we will use the above simple model. Hobbs et al. ${ }^{9}$ have performed both full-scale numerical simulations of the Kenics static mixer and the effect of problem parameters such as injection location on its performance. ${ }^{10}$ We hope that the tools developed here and tested on a simple model will be of help in future numerical studies of this sort.

As a result of the periodicity in the $z$ direction (assuming a mixer of infinite length), it is natural to consider a Poincaré map defined as follows. The cross-section to the flow is taken to be the beginning of a particular element of the mixer, and the Poincare map associates to each point in the cross-section its point at the end of the element of the mixer according to its motion along a fluid path line. We shall denote thus defined Poincaré map by $P$. In the flow there is a dimensionless parameter, $\beta$, which is referred to as the mixing strength. In Figs. 2(a) and 2(b) we show Poincaré maps for $\beta=2$ and $\beta=8$, respectively.

The exit age distribution is a quantity of interest in this system. It is the number of particles, plotted vertically, versus the time that they remain in the mixer, plotted horizontally. These plots were observed in Ref. 1 to be multipeaked. In Fig. 2 we also show isoresidence-time plots taken from
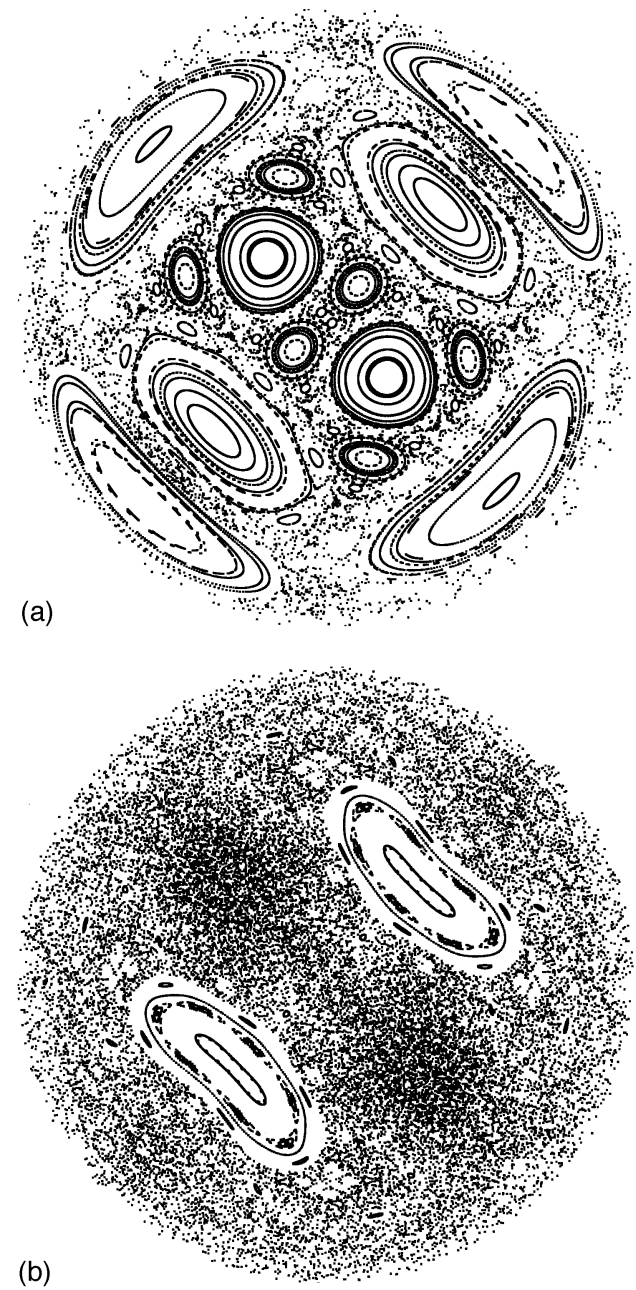

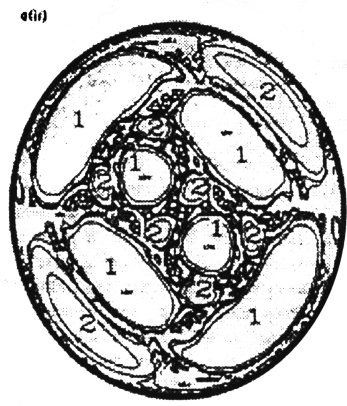

(c)

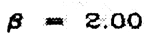

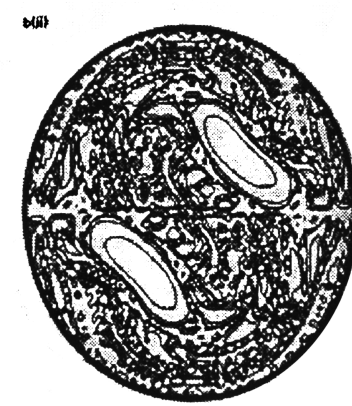

(d)

$\beta=8.00$
FIG. 2. Poincaré map for PPM at (a) $\beta=2$ and (b) $\beta=8$. Isoresidence times from Khakhar et al. at (c) $\beta=2$ and (d) $\beta=8$.

Khakhar et al. ${ }^{1}$ for (c) $\beta=2$ and (d) $\beta=8$. These are contours in the cross-section corresponding to points that remain in the mixer for the same amount of time.

Both the exit age distributions and isoresidence-time plots were computed integrating a uniform grid of points in the cross-section. Note the similarities between the Poincare sections (a) and (b) with the isoresidence-time plots (c) and (d) in Fig. 2. Khakhar et al. assert that the correspondence should be even closer as the length of the mixer increases. We will discuss these issues in terms of a new framework based on average velocities. Moreover, we will do this for a 
large class of unsteady duct flows described in the next section.

\section{Steady flows}

$$
\mathbf{v}\left(z_{1}, z_{2}, z_{3}\right)=\left(\dot{z}_{1}\left(z_{1}, z_{2}, z_{3}\right), \dot{z}_{2}\left(z_{1}, z_{2}, z_{3}\right), \dot{z}_{3}\left(z_{1}, z_{2}, z_{3}\right)\right)
$$

and $\mathbf{v}\left(z_{1}, z_{2}, z_{3}\right)$ in devices of the above described type are of the form

$$
\begin{aligned}
& \dot{z}_{1}=v_{1}\left(z_{1}, z_{2}, z_{3}\right), \\
& \dot{z}_{2}=v_{2}\left(z_{1}, z_{2}, z_{3}\right), \\
& \dot{z}_{3}=v_{3}\left(z_{1}, z_{2}, z_{3}\right),
\end{aligned}
$$

with $\mathbf{v}\left(z_{1}, z_{2}, z_{3}+l\right)=\mathbf{v}\left(z_{1}, z_{2}, z_{3}\right)$, where $l$ is the length of a basic periodic cell. The time that a particle spends crossing the cell is denoted by $\tau\left(z_{10}, z_{20}\right)$, where $\left(z_{10}, z_{20}\right)=\left[z_{1}(0)\right.$, $\left.z_{2}(0)\right]$ is the position in the cross-section of the particle starting from $z_{3}=0$ at time 0 . Note that $\tau$ is a function on the phase space of the Poincaré map described above, i.e., it associates a real number to each of the points in the crosssection $z_{3}=0$ of the pipe.

\section{B. Isoresidence-time plots and Poincaré maps}

The residence time of a particle starting at $\left(z_{10}, z_{20}, 0\right)$ at time $t=0$ in a pipe of length $L$ is given by

$$
\mathcal{T}^{L}\left(z_{10}, z_{20}\right)=\sum_{i=0}^{L / l-1} \tau\left(P^{i}\left(z_{10}, z_{20}\right)\right) .
$$

For very long pipes, we are interested in the rescaled, nondimensional version of the residence time, defined as

$$
\mathcal{T}_{r}^{L}=\frac{\mathcal{T}^{L}}{\bar{\tau} L / l}
$$

where $\bar{\tau}$ is the average of $\tau$ over the cross-section. Now we have

$$
\begin{aligned}
\lim _{L \rightarrow \infty} \mathcal{T}_{r}^{L}\left(z_{10}, z_{20}\right) & =\frac{1}{\bar{\tau}} \lim _{L \rightarrow \infty} \frac{l}{L} \sum_{i=0}^{L / l-1} \tau\left(P^{i}\left(z_{10}, z_{20}\right)\right) \\
& =\frac{\tau^{*}\left(z_{10}, z_{20}\right)}{\bar{\tau}}
\end{aligned}
$$

where

$$
\tau^{*}\left(z_{10}, z_{20}\right)=\lim _{n \rightarrow \infty} \frac{1}{n} \sum_{i=0}^{n-1} \tau\left(P^{i}\left(z_{10}, z_{20}\right)\right)
$$

is the time average of $\tau$ under the dynamics of $P$. Now, by Birkhoff's ergodic theorem, ${ }^{11} \tau^{*}$ is constant on the orbits of the Poincare map $P$. Thus for the limit of infinite length $L$ of the mixer, sets of constant rescaled residence times are composed of the orbits of the Poincare map $P$. To make numerical computation easier, we can present the above result in terms of the average velocity of particles. As

$$
l=\int_{0}^{\tau\left(z_{10}, z_{20}\right)} v_{3}\left(t, z_{10}, z_{20}\right) d t
$$

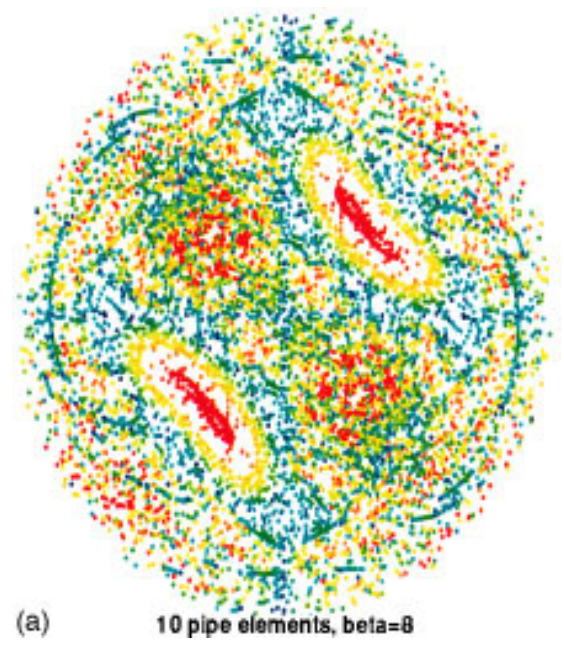

(a)

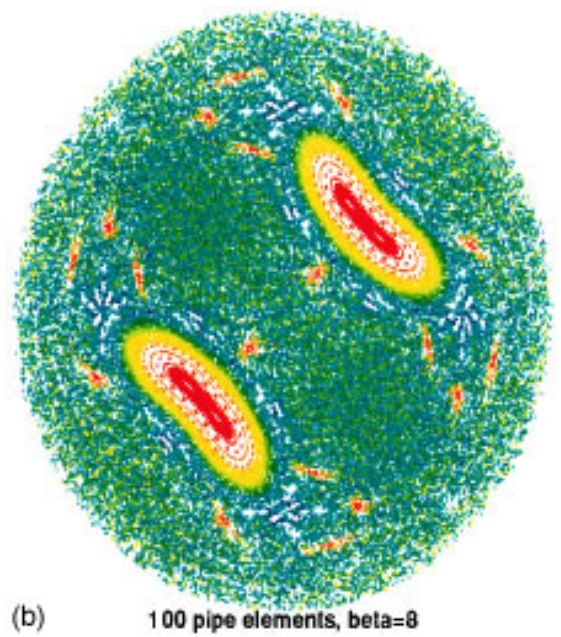

(b)

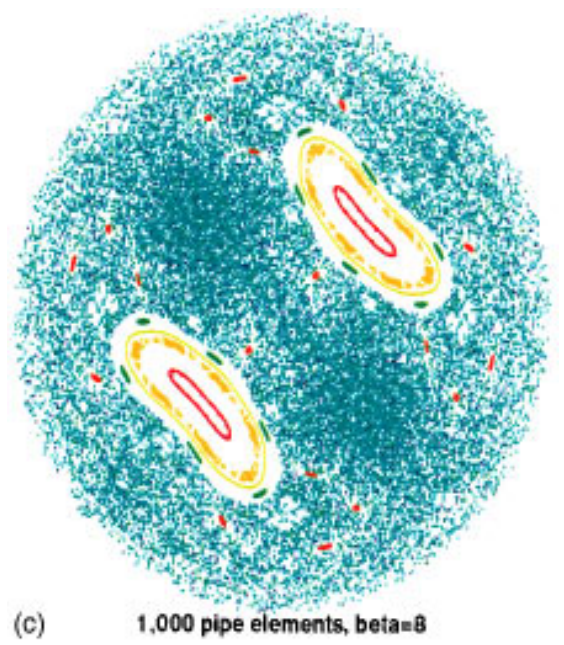

FIG. 3. The average velocities: (a) $\beta=8,10$ cells, (b) $\beta=8,100$ cells, (c) $\beta=8,1000$ cells.

where $v_{3}\left(t, z_{10}, z_{20}\right)$ is the axial velocity that a particle starting at $\left(z_{10}, z_{20}\right)$ has at time $t$, we obtain

$$
\tau\left(z_{10}, z_{20}\right)=\frac{l}{\frac{1}{\tau\left(z_{10}, z_{20}\right)} \int_{0}^{\tau\left(z_{10}, z_{20}\right)} v_{3}\left(t, z_{10}, z_{20}\right) d t},
$$

with the provision that when the denominator is zero, the left-hand side is defined to be infinity. Denoting by $\left\langle v_{3}\right\rangle$ 
$\times\left(z_{10}, z_{20}\right)$ the average velocity experienced by the particle starting at $\left(z_{10}, z_{20}\right)$ as it traverses the basic element of the mixer, we have

$$
\tau\left(z_{10}, z_{20}\right)=\frac{l}{\left\langle v_{3}\right\rangle\left(z_{10}, z_{20}\right)} .
$$

It is not hard to show that

$$
\tau^{*}\left(z_{10}, z_{20}\right)=\frac{l}{v_{3}^{*}\left(z_{10}, z_{20}\right)},
$$

where

$$
v_{3}^{*}\left(z_{10}, z_{20}\right)=\lim _{\bar{t} \rightarrow \infty} \frac{1}{\bar{t}} \int_{0}^{\bar{t}} v_{3}\left(t, z_{10}, z_{20}\right) d t
$$

is the time average of the axial velocity a particle would experience in an infinite pipe. Thus

$$
\begin{aligned}
\lim _{L \rightarrow \infty} \mathcal{T}_{r}^{L}\left(z_{10}, z_{20}\right) & =\frac{l}{\bar{\tau}}\left(\frac{1}{\left\langle v_{3}\right\rangle}\right)^{*}\left(z_{10}, z_{20}\right) \\
& =\frac{l}{\bar{\tau} v_{3}^{*}\left(z_{10}, z_{20}\right)}=\frac{\left\langle\bar{v}_{3}\right\rangle}{v_{3}^{*}\left(z_{10}, z_{20}\right)} .
\end{aligned}
$$

It is interesting to consider the change of the structure of the residence-time plots from $L / l=1$ to $L / l=\infty$. As shown above, this can be done by showing the plots of average velocities of particles after one basic element has been traversed, two basic elements have been traversed, etc. In Fig. 3(a) we show the plot of average velocities after 10 pipe lengths. It is clear that the average velocities are quite nonuniform and this indicates poor mixing. The features of the Poincaré map can already be seen. In Figs. 3(b) and 3(c) we show the velocity time-average plot after 100 and 1000 pipe lengths, respectively. The similarity with the Poincare map is clear, but these plots reveal that within the chaotic zone there are still zones where velocity averages are significantly different, colored blue. The points colored green have spent more time near the walls in the period studied, while the points colored blue have spent more time near the two big nonmixed islands. The difference in average velocities between the particles colored blue and those colored green shows how the average velocity plots give more information about mixing than the Poincaré maps. It is important to point out that the appropriate way of presenting these results is not through contour plots or density plots, as the chaotic nature of the data prevents efficient interpolation. We find that the best way to understand the process is to plot colored dots at the initial positions.

In Fig. 4 we show the average velocity histograms for the case $\beta=8,1000$ cells and $\beta=8,3000$ cells. It is seen that the nonuniformity of time averages in the chaotic zone is represented by the bimodal nature of the histogram around 0.1 for 1000 cells. When the number of cells is 3000 , the distribution becomes effectively unimodal and is centered around the mean value of the average velocity in the chaotic zone. However, there are still many small peaks in the histogram which are due to different, small chaotic zones.
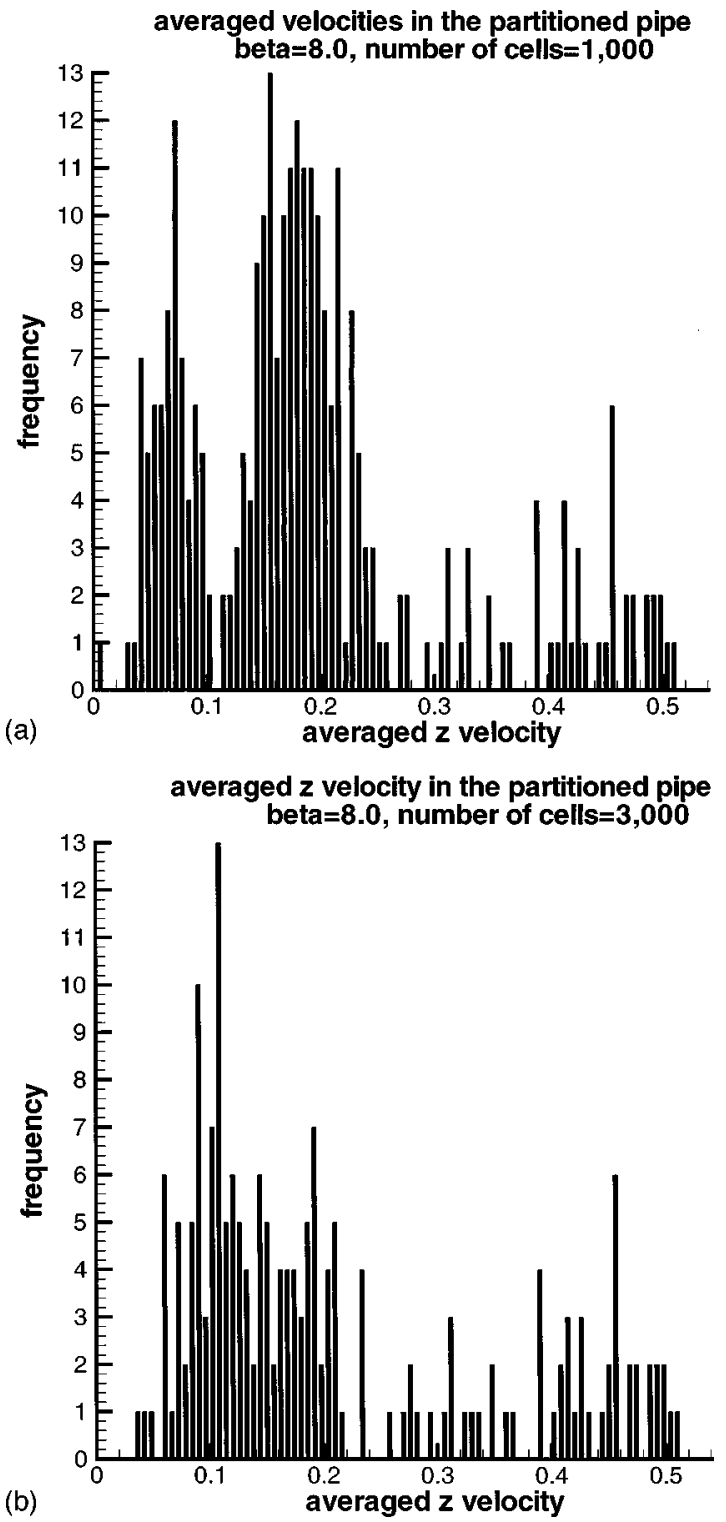

FIG. 4. Histogram of the average velocities: (a) $\beta=8,1000$ cells and (b) $\beta=8,3000$ cells.

The average velocity plot is shown in Fig. 5 for $\beta=2$, 1000 cells. It seems that the average velocity in the chaotic zone is uniform and thus mixing is good there. Of course, the existence of large islands prevents good mixing throughout the cross-section in this case. While it is obvious that mixing is worse in this case, the question arises of whether the overall mixing property of the flow can be characterized by a single number. This question was addressed in Refs. 12 and 13 , where it was shown that the efficiency of mixing of different flows can be measured using Kolmogorov-Sinai entropy. Subsequent computations showed that $\beta=2$ case indeed has lower Kolmogorov-Sinai entropy that $\beta=8$ case.

\section{UNSTEADY, SYMMETRIC FLOWS IN PIPES}

In this section we will develop a theory of residence times for unsteady flows in pipes that is analogous to the one that we have developed in the previous section for steady flows periodic along the axis of a pipe. 


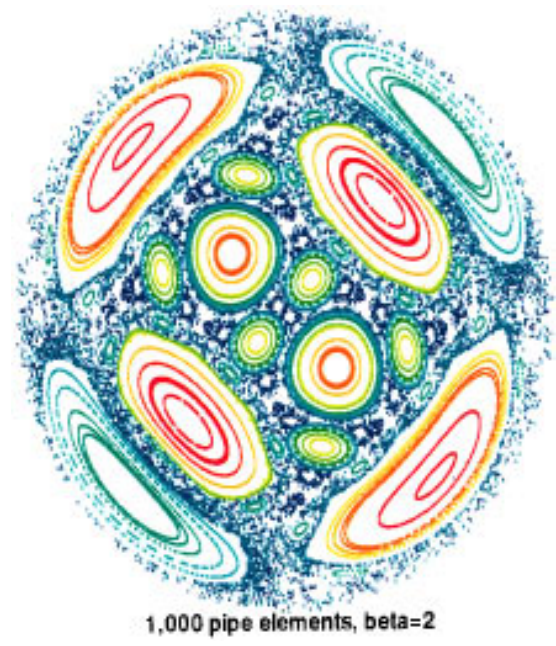

FIG. 5. The average velocities, $\beta=2,1000$ cells

\section{A. Quasiperiodic flows}

Consider a laminar, time-dependent flow in a pipe of arbitrary shape (see Fig. 6).

We assume that the velocity is independent of the variable running along the axis of the pipe. For this, some symmetry of the pipe geometry is necessary; see Ref. 14. As shown in Ref. 14 such a symmetry implies that the threedimensional flow has a stream function, and that coordinates $z_{1}, z_{2}, z_{3}$ can be found so that in these new coordinates the flow can be written as

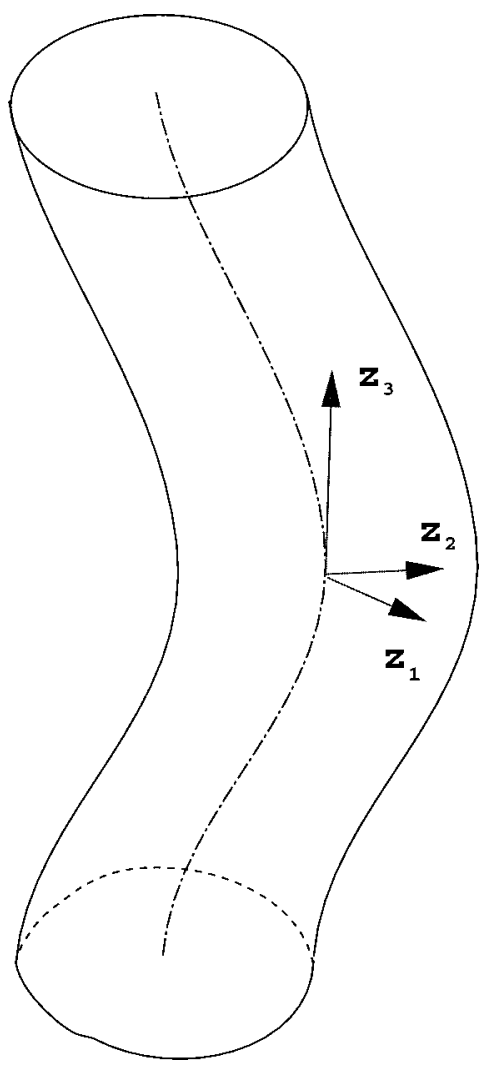

FIG. 6. Geometry of the curved pipe.

$$
\begin{aligned}
& \dot{z}_{1}=-\frac{\partial \psi}{\partial z_{2}}\left(z_{1}, z_{2}, t\right), \\
& \dot{z}_{2}=\frac{\partial \psi}{\partial z_{1}}\left(z_{1}, z_{2}, t\right), \\
& \dot{z}_{3}=f\left(z_{1}, z_{2}, t\right) .
\end{aligned}
$$

For example, in the case of helical symmetry we would have $z_{1}=r^{2}, z_{2}=\theta-z, z_{3}=z$, where $r, \theta, z$ are the standard cylindrical coordinates. ${ }^{15}$ Franjione and Ottino ${ }^{16}$ refer to a steady version of Eq. (5) as duct flows. It can be seen that the first two equations in Eq. (5) decouple from the third (i.e., the dynamics of the first two equations is unaffected by $z_{3}$ ). As is well-known, the dynamics of fluid particles in the cross-section of the pipe, described by the first two equations in the unsteady duct flow (5), can be chaotic since they have the form of a one and a half degree of freedom Hamiltonian system. The dynamics of the third variable depends entirely on the dynamics of $z_{1}$ and $z_{2}$, which are bounded. The $z_{3}$ coordinate may be unbounded, or the flow could be periodic in $z_{3}$.

The nature of the time dependence of Eq. (5) will be important for our analysis. Generally, the techniques we develop can be applied to velocity fields having quasiperiodic time dependence. By this we mean that Eq. (5) can be written in the form

$$
\begin{aligned}
& \dot{z}_{1}=-\frac{\partial \psi}{\partial z_{2}}\left(z_{1}, z_{2}, \omega_{1} t, \ldots, \omega_{n} t\right), \\
& \dot{z}_{2}=\frac{\partial \psi}{\partial z_{1}}\left(z_{1}, z_{2}, \omega_{1} t, \ldots, \omega_{n} t\right), \\
& \dot{z}_{3}=f\left(z_{1}, z_{2}, \omega_{1} t, \ldots, \omega_{n} t\right),
\end{aligned}
$$

where $\omega_{1}, \ldots, \omega_{n}$ are $n$ frequencies, and each component of Eq. (6) is periodic in the arguments $\omega_{i} t$, with periods $2 \pi / \omega_{i}$, for $i=1, \ldots, n$.

Our analysis is more conveniently carried out in the context of an associated Poincaré map. In constructing this map it is useful to employ a standard dynamical systems "trick" that expresses Eq. (6) as a time independent velocity field through enlarging the set of differential equations by introducing the phases of the different frequency components as new variables. In this case Eq. (6) becomes

$$
\begin{aligned}
& \dot{z}_{1}=-\frac{\partial \psi}{\partial z_{2}}\left(z_{1}, z_{2}, \theta_{1}, \ldots, \theta_{n}\right), \\
& \dot{z}_{2}=\frac{\partial \psi}{\partial z_{1}}\left(z_{1}, z_{2}, \theta_{1}, \ldots, \theta_{n}\right), \\
& \dot{z}_{3}=f\left(z_{1}, z_{2}, \theta_{1}, \ldots, \theta_{n}\right), \\
& \dot{\theta}_{1}=\omega_{1}, \\
& \vdots \\
& \dot{\theta}_{n}=\omega_{n} .
\end{aligned}
$$

Since $\theta_{i}(t)=\omega_{i} t+\theta_{i 0}, i=1, \ldots, n$, we see that Eq. (7) is equivalent to Eq. (6). We construct the Poincaré map using 
Eq. (7). Consider any frequency component, say $\omega_{n}$ (otherwise, re-index the frequencies), with associated period $T$ $=2 \pi / \omega_{n}$. A cross-section to Eq. (7) is

$$
\Sigma \equiv\left\{\left(z_{1}, z_{2}, z_{3}, \theta_{1}, \ldots, \theta_{n-1}, \theta_{n}\right) \mid \theta_{n}=0\right\} .
$$

In order to make the formulae less cumbersome, we introduce the following notation:

$$
\begin{aligned}
& z \equiv\left(z_{1}, z_{2}, z_{3}\right), \quad z_{0} \equiv\left(z_{10}, z_{20}, z_{30}\right), \\
& \theta \equiv\left(\theta_{1}, \ldots, \theta_{n-1}\right), \quad \theta_{0} \equiv\left(\theta_{10}, \ldots, \theta_{(n-1) 0}\right), \\
& \omega \equiv\left(\omega_{1}, \ldots, \omega_{n-1}\right) .
\end{aligned}
$$

The Poincaré map, denoted $M$, of $\Sigma$ into itself is then defined as

$$
\begin{aligned}
& M: \Sigma \rightarrow \Sigma, \\
& \left(z_{0}, \theta_{0}\right) \mapsto\left(z\left(T, z_{0}, \theta_{0}\right), \theta_{0}+2 \pi \frac{\omega}{\omega_{n}}\right),
\end{aligned}
$$

where $z\left(t, z_{0}, \theta_{0}\right)$ is the solution of the $z_{1}-z_{2}-z_{3}$ component of Eq. (7) through the point $\left(z_{0}, \theta_{0}\right)$ at $t=0$. In other words, the computation of the Poincare map involves fixing one frequency and finding the position of the particle after one period in that frequency.

We will mainly be interested in the motion of particles down the pipe. The $z_{3}$ component of the Poincare map is given by

$$
z_{3}\left(T, z_{0}, \theta_{0}\right)=z_{30}+\int_{0}^{T} f\left(z\left(t, z_{0}, \theta_{0}\right), \omega t+\theta_{0}, \omega_{n} t\right) d t,
$$

and the displacement in the $z_{3}$ direction after $n+1$ discrete time steps is given by

$$
z_{3}^{n+1}=z_{3}^{n}+j\left(z^{n}, \theta^{n}\right),
$$

where

$$
j\left(z^{n}, \theta^{n}\right) \equiv \int_{0}^{T} f\left(z\left(t, z^{n}, \theta^{n}\right), \omega t+\theta^{n}, \omega_{n} t\right) d t
$$

denotes the "jump" in the $z$ direction after each discrete time step. From this expression, and the definition of the Poincaré map $M$, it is easy to see that

$$
\begin{aligned}
z_{3}^{n+1} & =F^{n}\left(z_{10}, z_{20}, \theta_{0}\right), \\
& =\sum_{k=0}^{n-1} j\left(z_{1}^{k}, z_{2}^{k}, \theta^{k}\right)=\sum_{k=0}^{n-1} j\left(M^{k}\left(z_{0}, \theta_{0}\right)\right) ;
\end{aligned}
$$

this notation will be used throughout the rest of this paper.

We want to point out an important property of Eq. (12). In particular, it is only a function of the variables $z_{10}, z_{20}$, and $\theta_{0}$. The $z_{30}$ variable only enters as a parameter. This is because the $z_{3}$ component of the velocity field $(f)$ depends only on $z_{1}, z_{2}$, and $t$. This is important since we will want to apply the Birkhoff ergodic theorem to the function $j$, which will require $j$ to be defined on a bounded domain (which is simply the cross-sectional variables of the pipe and the phases of the frequency components).

\section{B. Isoresidence-time plots and Poincaré maps}

Here we derive a relationship between isoresidence-time plots and Poincaré maps of unsteady duct flows, and provide an approximate method of calculation of residence time which shortens the calculation time significantly.

We will assume that $z_{30}$ is the same for all the particles. Let $j^{*}$ denote the time average of $j$, defined by

$$
j^{*}\left(z_{10}, z_{20}, \theta_{0}\right)=\lim _{n \rightarrow \infty} \frac{F^{n}\left(z_{10}, z_{20}, \theta_{0}\right)}{n} .
$$

The quantity $\bar{j}$ is defined as

$$
\bar{j}=\int j d z_{10} d z_{20} d \theta_{0}
$$

and let $\langle j\rangle$ denote the space average of $j$ defined by

$$
\langle j\rangle=\int j p d z_{10} d z_{20} d \theta_{0},
$$

where $p=p\left(z_{10}, z_{20}, \theta_{0}\right)$ denotes the initial distribution of points.

We shall consider the case when $\left|j^{*}\left(z_{10}, z_{20}, \theta_{0}\right)\right|>c$ $>0$, and $j$ bounded. Let $N^{L}\left(z_{10}, z_{20}, \theta_{0}\right)$ be the first passage time (residence time, first exit time) for the point $\left(z_{10}, z_{20}, \theta_{0}\right)$, i.e.,

$$
z_{3}^{n}>L
$$

where $L$ is the total length of the pipe. At iterate $n$ $=N^{L}\left(z_{10}, z_{20}, \theta_{0}\right)$ and $z_{3}^{n}<L$ for every $n$ less than $N^{L}\left(z_{10}, z_{20}, \theta_{0}\right)$. Consider the renormalized residence time

$$
N_{r}^{L}\left(z_{10}, z_{20}, \theta_{0}\right)=\frac{N^{L}\left(z_{10}, z_{20}, \theta_{0}\right) \bar{j}}{L} .
$$

The main result of this section is the following

$$
\lim _{L \rightarrow \infty} N_{r}^{L}\left(z_{10}, z_{20}, \theta_{0}\right)=\frac{\bar{j}}{j^{*}\left(z_{10}, z_{20}, \theta_{0}\right)} .
$$

This formula has interesting consequences, which we now discuss.

By Birkhoff's ergodic theorem (see, e.g., Ref. 11), $j^{*}\left(z_{10}, z_{20}, \theta_{0}\right)$ must be constant on the orbits of the crosssectional Poincaré map $M$. Therefore, isoresidence sets on which $N_{r}^{L}$ is constant are, for the limit of an infinite pipe, composed of orbits of the cross-sectional Poincare map $M$. It also follows from this result that almost all the points in the chaotic region of the cross-sectional Poincare map will have the same residence time since in these regions $M$ can be assumed to be ergodic, and so $j^{*}\left(z_{10}, z_{20}, \theta_{0}\right)$ is constant there.

This result is actually quite a practical tool. Residencetime plots are useful in a variety of applications in chemical engineering, mechanical engineering and elsewhere. Before designing a long pipe, one would like to know what the residence times for a particular device (heat exchanger, mixer) will be. Typically, in complex geometries, analytical models are not available. So, isoresidence-time sets are com- 
puted numerically. For long devices this requires a lot of computer time, as the time to get out of the pipe becomes very long.

Consider a singly periodic flow with frequency $\omega$. Let us define the axial Strouhal number, $S_{a x}$ by

$$
S_{a x}=\frac{\omega L}{2 \pi U_{a x}},
$$

where $U_{a x}$ is the characteristic axial velocity. Note that we can define $S_{a x}$ as

$$
S_{a x}=\frac{L}{j^{*}}
$$

so that it represents the average residence time (i.e., the average number of iterations of the Poincare map it takes to exit the pipe). The cross-sectional Strouhal number $S_{c s}$ can be defined as

$$
S_{c s}=\frac{\omega R}{2 \pi U_{c s}},
$$

where $U_{c s}$ is the characteristic cross-sectional velocity, and $R$ the radius of the pipe. $S_{c s}$ represents the average number of iterations of the Poincare map it takes to traverse the crosssection of the pipe. Now assume $S_{c s} \ll S_{a x}$. This condition will assure that the cross-section is explored thoroughly by fluid particles in only a fraction of the average time necessary to exit the device. They are to be met easily if the flow is laminar so characteristic velocities are small, $U_{a x}$ and $U_{c s}$ are of the same magnitude, and $L \gg R$. The situation is even more favorable if $U_{a x} \ll U_{c s}$. Because of these conditions, a well-resolved cross-sectional Poincaré map can be obtained in a time that is short compared with the average exit time. Thus regions of chaotic behavior and regions of regular behavior can be uncovered. As the motion in the chaotic zones is assumed to be ergodic, the time average of $j$ there can be obtained by integrating $j$ over the area of the chaotic zone, i.e.,

$$
j^{*}=\int_{A_{\text {chaotic }}} j\left(z_{10}, z_{20}, \theta_{0}\right) d z_{10} d z_{20} d \theta_{0} .
$$

This time average is constant throughout the chaotic zone. The exit time for any initial condition in that zone for long pipe is given approximately by

$$
\begin{aligned}
N^{L}\left(z_{10}, z_{20}, \theta_{0}\right) & \approx \frac{L}{j^{*}\left(z_{10}, z_{20}, \theta_{0}\right)} \\
& =\frac{L}{\int_{A_{\text {chaotic }}} j\left(z_{10}, z_{20}, \theta_{0}\right) d z_{10} d z_{20} d \theta_{0}} .
\end{aligned}
$$

The time averages of $j$ on regular orbits can be obtained by taking the average of $j$ over the orbit.

We now give a proof of Eq. (13). We need to show that for any sequence of pipe lengths $\left\{L_{i}\right\}$, such that $L_{i} \rightarrow \infty$ when $i \rightarrow \infty$, for every $\epsilon>0$ there exists $I$ such that $i \geqslant I$ implies

$$
\left|N_{r}^{L_{i}}\left(z_{10}, z_{20}, \theta_{0}\right)-\frac{\bar{j}}{j^{*}\left(z_{10}, z_{20}, \theta_{0}\right)}\right|<\epsilon .
$$

So, pick such a sequence, $\left\{L_{i}\right\}$, and $\epsilon$. Note that we can always find $\zeta_{2}\left(z_{10}, z_{20}, \theta_{0}, L_{i}\right)$ such that

$$
F^{N^{L}\left(z_{10}, z_{20}, \theta_{0}\right)}\left(z_{10}, z_{20}, \theta_{0}\right)+\zeta_{2}\left(z_{10}, z_{20}, \theta_{0}, L_{i}\right)=L_{i},
$$

where $\zeta_{2}$ is defined by the above equation. $\zeta_{2}$ is bounded for every integer $i$, and almost every initial condition, as

$$
\begin{aligned}
& F^{N^{L_{i}\left(z_{10}, z_{20}, \theta_{0}\right)-1}\left(z_{10}, z_{20}, \theta_{0}\right)} \\
& <L_{i} \leqslant F^{N^{L} i\left(z_{10}, z_{20}, \theta_{0}\right)}\left(z_{10}, z_{20}, \theta_{0}\right) \text {, }
\end{aligned}
$$

so

$$
\zeta_{2}\left(z_{10}, z_{20}, \theta_{0}, L_{i}\right) \leqslant \max _{\left(z_{10}, z_{20}, \theta_{0}\right)} j\left(z_{10}, z_{20}, \theta_{0}\right) .
$$

Now, by the Birkhoff ergodic theorem,

$$
\begin{aligned}
& F^{N^{L}\left(z_{10}, z_{20}, \theta_{0}\right)}\left(z_{10}, z_{20}, \theta_{0}\right) \\
& \quad=N^{L_{i}}\left(z_{10}, z_{20}, \theta_{0}\right) j^{*}\left(z_{10}, z_{20}, \theta_{0}\right)+\zeta_{1}\left(z_{10}, z_{20}, \theta_{0}, L_{i}\right),
\end{aligned}
$$

for almost every initial condition, where

$$
\lim _{i \rightarrow \infty} \frac{\zeta_{1}\left(z_{10}, z_{20}, \theta_{0}, L_{i}\right)}{N^{L_{i}}\left(z_{10}, z_{20}, \theta_{0}\right)}=0 .
$$

Let

$$
\zeta=\zeta_{1}+\zeta_{2}
$$

Now, for every $\delta>0$, we can choose $I$ such that

$$
\left|\frac{\zeta\left(z_{10}, z_{20}, \theta_{0}, L_{i}\right)}{N^{L_{i}\left(z_{10}, z_{20}, \theta_{0}\right)}}\right|<\delta,
$$

for every $i>I$. Choose

$$
\delta<\min \left(\left|j^{*}\right|, \frac{\epsilon\left(j^{*}\right)^{2}}{\bar{j}+\epsilon\left|j^{*}\right|}\right)
$$

Equations (14), (15) and (16) now can be used to show that 


$$
\begin{aligned}
\left|N_{r}^{L_{i}}\left(z_{10}, z_{20}, \theta_{0}\right)-\frac{\bar{j}}{j^{*}\left(z_{10}, z_{20}, \theta_{0}\right)}\right| \\
=\left|\frac{N^{L_{i}\left(z_{10}, z_{20}, \theta_{0}\right) \bar{j}}}{L_{i}}-\frac{\bar{j}}{j^{*}\left(z_{10}, z_{20}, \theta_{0}\right)}\right| \\
=\mid \frac{N^{L_{i}\left(z_{10}, z_{20}, \theta_{0}\right) \bar{j}}}{N^{L_{i}\left(z_{10}, z_{20}, \theta_{0}\right) j^{*}+\zeta\left(z_{10}, z_{20}, \theta_{0}, L_{i}\right)}} \\
=\left|\frac{\bar{j}}{j^{*}\left(z_{10}, z_{20}, \theta_{0}\right)}\right| \\
=\left|\frac{\zeta\left(z_{10}, z_{20}, \theta_{0}, L_{i}\right)}{N^{L_{i}\left(z_{10}, z_{20}, \theta_{0}\right)} \bar{j}}\right|<\left|\frac{\bar{j} \delta}{\left(j^{*}\right)^{2}-\delta j^{*}}\right|<\epsilon,
\end{aligned}
$$

for every $i>I$. The above argument is valid, by Birkhoff's ergodic theorem, for almost every initial condition $\left(z_{10}, z_{20}, \theta_{0}\right)$. Thus our proof is complete.

\section{AXIAL DISTRIBUTION OF PARTICLES}

In the previous section, we have analyzed residence times of particles in a class of unsteady duct flows. Another question is natural in this context: what is the distribution of particles along the axis when time is very large. As we noted earlier, it was observed by Khakhar et al. ${ }^{1}$ that such a distribution can be multimodal for some steady chaotic flows in pipes. Here we investigate this question in the context of unsteady duct flows.

We assume that all particles start at $z_{3}=0$ at time $t=0$, and their initial distribution is defined by some density $p\left(z_{10}, z_{20}, \theta_{0}\right)$, where

$$
\int_{A} p\left(z_{10}, z_{20}, \theta_{0}\right) d z_{10} d z_{20} d \theta_{0}=1,
$$

and $A$ denotes some subset of the flow domain. The distribution function that gives us the amount of particles that are, at step $n$ of the iteration, below or at some $Z_{3}$ is defined as

$$
W^{n}\left(Z_{3}\right)=P\left(\left\{\left(z_{10}, z_{20}, \theta_{0}\right) \mid F^{n}\left(z_{10}, z_{20}, \theta_{0}\right) \leqslant Z_{3}\right\}\right),
$$

where $P$ denotes a probability measure defined as follows. If we denote the set of initial conditions $\left\{\left(z_{10}, z_{20}\right.\right.$, $\left.\left.\theta_{0}\right) \mid F^{n}\left(z_{10}, z_{20}, \theta_{0}\right) \leqslant Z_{3}\right\}$ by $A_{Z_{3}}$, then $P\left(A_{Z_{3}}\right)$ is just

$$
P\left(A_{Z_{3}}\right)=\int_{A_{Z_{3}}} p\left(z_{10}, z_{20}, \theta_{0}\right) d z_{10} d z_{20} d \theta_{0},
$$

i.e., the integral over all the initial conditions for which the axial position after $n$ steps is below $Z_{3}$. We need to somehow rescale the variable $Z_{3}$ in this distribution, as in the case of a large length of pipe; most of the particles will move toward infinity along the axis. The exception might be those with zero average velocity. Let us consider the situation in which particles do not sample the whole cross-section (this is typical: for example, KAM tubes can prevent completeness of mixing in the cross-section, although the cross-sectional flow is ergodic in some zones). Thus the time average of the axial velocity is not going to be the same for all particles (if the axial flow is not plug flow). An appropriate scaling is

$$
Y=\frac{Z_{3}-\bar{j}^{*} n}{\sigma(n)},
$$

where

$$
\sigma^{2}(n)=\left\langle\left(F^{n}-\left\langle F^{n}\right\rangle\right)^{2}\right\rangle .
$$

This is a common rescaling: subtract the mean distance from the origin, and divide by the size of the cloud of particles (i.e., standard deviation). In the case under consideration here, $\sigma(n)$ satisfies

$$
\lim _{n \rightarrow \infty} \frac{\sigma^{2}(n)}{n^{2}}=\left\langle\left(j^{*}-\left\langle j^{*}\right\rangle\right)^{2}\right\rangle \stackrel{\operatorname{def}}{=} a,
$$

so

$$
\sigma(n) \sim n
$$

for large times $n$. This is due to nonuniform time-averaged velocities of particles. ${ }^{17,18}$ It is clear from Eq. (18) that $Y$ is centered at $z_{3}=\bar{j}^{*} n$, so it moves with the "velocity" $j$ averaged over time and the initial conditions. Now,

$$
\begin{aligned}
W^{n}\left(Z_{3}\right) & =P\left\{\left(z_{10}, z_{20}, \theta_{0}\right) \mid F^{n}\left(z_{10}, z_{20}, \theta_{0}\right) \leqslant Z_{3}\right\} \\
& =P\left\{\left(z_{10}, z_{20}, \theta_{0}\right) \mid F^{n}\left(z_{10}, z_{20}, \theta_{0}\right) \leqslant \sigma Y+\bar{j}^{*} n\right\} \\
& =P\left\{\left(z_{10}, z_{20}, \theta_{0}\right) \mid \frac{F^{n}\left(z_{10}, z_{20}, \theta_{0}\right)-\bar{j}^{*} n}{\sigma} \leqslant Y\right\} \\
& \stackrel{\operatorname{def}}{=} G^{n}(Y) .
\end{aligned}
$$

We shall call the above defined distribution $G^{n}$ the renormalized distribution. Just like any genuine distribution, it is zero at negative infinity, as no particles are there at any finite time, and 1 at positive infinity, as all particles are below positive infinity at any finite time. As opposed to $W^{n}$, it has interesting asymptotic properties when $n$ is very large. Consider yet another distribution, that we will call $G^{\infty}$, defined by

$$
G^{\infty}(Y)=P\left\{\left(z_{10}, z_{20}, \theta_{0}\right) \mid \frac{j^{*}\left(z_{10}, z_{20}, \theta_{0}\right)-\bar{j}^{*}}{\sqrt{a}} \leqslant Y\right\} .
$$

It can be shown ${ }^{17}$ that the distribution $G^{n}$ approaches $G^{\infty}$ when $n$ goes to infinity. So, let us discuss some properties of $G^{\infty}$. First, we have

$$
G^{\infty}(Y)=P\left(A_{Y}\right)=\int_{A_{Y}} p\left(z_{10}, z_{20}, \theta_{0}\right) d z_{10} d z_{20} d \theta_{0},
$$

where $A(Y)$ is the set of all initial conditions in the crosssection such that 


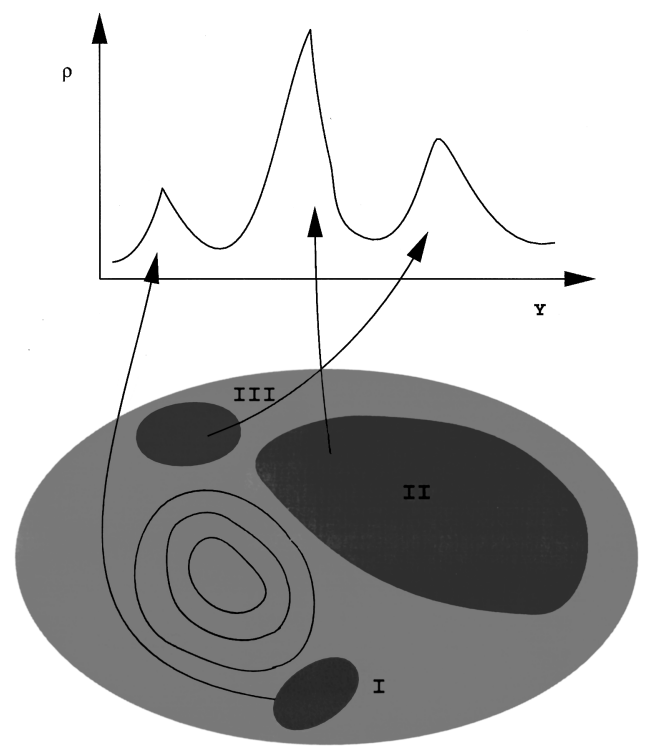

FIG. 7. Chaotic zones in the cross-section contribute peaks to the probability density of particles.

$$
\frac{j^{*}\left(z_{10}, z_{20}, \theta_{0}\right)-\bar{j}^{*}}{\sqrt{a}} \leqslant Y .
$$

Now assume that $Y_{j}$ is such that the corresponding value of $j^{*}\left(z_{10}, z_{20}, \theta_{0}\right)=\sqrt{a} Y_{j}+\bar{j}^{*}$ is uniform in a nonzero area chaotic zone of the Poincare map (because the motion is supposedly ergodic there). Furthermore, assume for simplicity that the initial distribution is uniform over the crosssection. Thus as can be seen from Eq. (22), $G^{\infty}$ will have a jump of the size

$$
\int_{A_{Y_{j}}} d z_{10} d z_{20} d \theta_{0}
$$

at $Y_{j}$, where $A_{Y_{j}}$ is the chaotic zone. Thus we have found that chaotic zones cause discontinuities in asymptotic distribution functions.

The results of experiments are often presented in terms of histograms. ${ }^{1}$ In the standard probability theory formalism, this would correspond to a function $\rho(Y)$, such that

$$
G^{\infty}\left(Y_{2}\right)-G^{\infty}\left(Y_{1}\right)=\int_{Y_{1}}^{Y_{2}} \rho(Y) d Y
$$

for any $Y_{1}, Y_{2}$, i.e., $\rho(Y)$ is a probability density for the particle distribution. Any discontinuity at $Y_{j}$ in $G^{\infty}$ must be caused by a $\delta$-function behavior of $\rho(Y)$. For finite $n$, the peak is smoothened out, and thus appears as an extremum of the probability density. If there is more than one chaotic zone for the cross-sectional Poincaré map, this will cause more than one extremum of the probability density, i.e., multimodality.

This situation is shown in Fig. 7. We assume that three isolated, in the sense that the particles do not cross over between these zones, chaotic zones exist, denoted by I, II and III. Each of the three isolated chaotic zones contributes a peak to the probability density function.

\section{CONCLUSIONS}

We have derived some rigorous results about the relationship of Poincaré maps and residence-time distributions in flows exhibiting chaotic advection. It has been only recently, both within industrial research laboratories and at universities, that the importance of a detailed study of residence-time distributions in laminar mixers has been realized. Indeed, the Lagrangian properties of flows in laminar mixers can be very complicated. The appropriate statistical theory for these flows is not probability theory, but ergodic theory of deterministic maps and flows.

Velocity time-average plots (or, equivalently, residencetime plots) reveal a lot about the statistical structure of the particle motion in pipes and mixers. Our numerical results on the partitioned-pipe mixer show that a color residence-time plot can indicate the nonuniformity of mixing in a zone in which the Poincaré map shows complete mixing. In relation to this, the coexistence of nonmixed islands and solid boundaries in the pipe flow was shown to introduce multimodality of finite-time particle distributions along the axis. Another source of multimodality is the coexistence of different chaotic zones in the cross-section. This theory can also provide an explanation for the observed steep gradients in residencetime distributions observed in experiments by Saxena and Nigam. ${ }^{4}$ In their apparatus with helically coiled cells, steeper gradients have been observed when more cells were present. We have shown that this corresponds to the uniformization of time averages within a chaotic zone in the cross-section.

There are not many experimental studies of the matter under analysis here, except for the one by Kusch and Ottino $^{19}$ who studied flows of the type we have considered.

In this paper we have used some of the basic facts of ergodic theory, such as the existence of velocity timeaverages along particle paths. We are convinced that the use of ergodic theory in this context is far beyond the results obtained here.

\section{ACKNOWLEDGMENTS}

This research was partially supported by ONR. Grant No. N00014-98-1-0056 and AFOSR Grant No. F49620-971-0293 to I.M. and ONR Grant No. N00014-97-1-0071 to S.W.

${ }^{1}$ D. V. Khakhar, J. G. Franjione, and J. M. Ottino, “A case study of chaotic mixing in deterministic flows: the partitioned pipe mixer," Chem. Eng. Sci. 42, 2909-2926 (1987).

${ }^{2}$ J. C. R. Turner, "Perspectives in residence-time distributions," Chem. Eng. Sci. 38, 1-4 (1983).

${ }^{3}$ P. V. Danckewerts, "Continuous flow systems," Chem. Eng. Sci. 2, 1-13 (1953).

${ }^{4}$ A. K. Saxena and K. D. P. Nigam, "Coiled configuration for flow inversion and its effect on residence time distribution," AIChE J. 30, 363-368 (1984).

${ }^{5}$ J. M. Ottino, The Kinematics of Mixing: Stretching, Chaos and Transport (Cambridge University Press, Cambridge, 1989).

${ }^{6}$ J. H. Seinfeld and L. Lapidus, Mathematical Methods in Chemical Engineering (Prentice-Hall, Englewood Cliffs, 1974).

${ }^{7}$ H. Aref, "Stirring by chaotic advection," J. Fluid Mech. 143, 1-21 (1984).

${ }^{8}$ O. Byrde and M. L. Sawley, "Gaining insights into fluid mixing via massively parallel flow computations," Technical report, Fluid Mechanics Laboratory, Ecole Polytechnique Fédérale de Laussane (1998). 
${ }^{9}$ D. M. Hobbs, P. D. Swanson, and F. J. Muzzio, “'Numerical characterization of low Reynolds number flow in the Kenics static mixer," Chem. Eng. Sci. 53, 1565-1584 (1997).

${ }^{10}$ D. M. Hobbs and F. J. Muzzio, "Effects of injection location, flow ratio and geometry on Kenics mixer performance,' AIChE. J. 43, 3121-3132 (1997).

${ }^{11}$ K. Petersen, Ergodic Theory (Cambridge University Press, Cambridge, 1995).

${ }^{12}$ D. D'Alessandro, M. Dahleh, and I. Mezić, "Control of mixing in fluid flows: A maximum entropy approach,' IEEE Trans. Autom. Control. (to be published).

${ }^{13}$ D. D’Alessandro, M. Dahleh, and I. Mezić, "'Measuring mixing using Kolmogorov-Sinai entropy,' UCSB preprint (1998).
${ }^{14}$ I. Mezić and S. Wiggins, "On the integrability and perturbation of three dimensional fluid flows with symmetry,' J. Nonlinear Sci. 4, 157-194 (1994).

${ }^{15}$ I. Mezić, A. Leonard, and S. Wiggins, "Regular and chaotic particle motion near a helical vortex filament,', Physica D 111, 179-201 (1998).

${ }^{16}$ J. G. Franjione and J. M. Ottino, 'Stretching in duct flows,', Phys. Fluids A 3, 2819-2821 (1991).

${ }^{17}$ I. Mezić, Ph.D. thesis, California Institute of Technology, 1994.

${ }^{18} \mathrm{I}$. Mezic and S. Wiggins, "On the dynamical origin of asymptotic $t^{2}$ dispersion of a nondiffusive tracer in incompressible laminar flows," Phys. Fluids A 6, 2227-2229 (1995).

${ }^{19}$ H. A. Kusch and J. M. Ottino, "Experiments on mixing in continuous flows," J. Fluid Mech. 236, 319-348 (1992). 